\title{
BURNOUT, SATISFACCIÓN LABORAL Y AUTONOMÍA LABORAL COMO DETERMINANTES DE INTENCIÓN DE ABANDONO Y RETENCIÓN LABORAL DE ENFERMERAS Y AUXILIARES DE ENFERMERÍA
}

\author{
Olga Garrido \\ Enfermera Matrona, Magister en Administración de Salud, Pontificia Universidad Católica de Chile. \\ Gladys Maulen \\ Kinesióloga, Magister en Administración de Salud, Pontificia Universidad Católica de Chile. \\ Andrés Raineri \\ Psicólogo, Ph.D. Facultad de Economía y Administración, Pontificia Universidad Católica de Chile. \\ e-mail: araineri@uc.cl | Vicuña Mackenna 4860, Macul, Santiago, Chile | Tel: 26864367
}

\begin{abstract}
This study analyzes the antecedents of intentions to leave, and stay in the organization, in a sample of nurses and nurse aides by means of a survey methodology. Results suggest that higher levels of burnout, lower levels satisfaction with pay, and lower levels of work autonomy are associated with intent to leave both in nurses and nurse aides. Furthermore, in the case of nurses', the flexibility of their work shift schedule explains additional variance of intention to leave. Increasing age explains additional variance in nurse aides' intention to leave. Intentions to remain in the organization are explained by schedule flexibility and autonomy at work in the case of nurses, and lower levels of emotional exhaustion and higher levels of fulfillment at work in the case of nurse aides.
\end{abstract}

Key words: Burnout; Work Satisfaction; Job Autonomy; Intention to Leave

\begin{abstract}
RESUMEN
Este estudio efectúa un análisis de las variables antecedentes de las intenciones de abandono laboral, y de permanencia en la organización, de una muestra de enfermeras y auxiliares de enfermería usando una metodología de encuestas. Los resultados indican que mayores niveles de burnout, menor satisfacción con remuneraciones, y menor autonomía en el trabajo se asocian a intención de abandono de enfermeras y auxiliares de enfermería. Además, en el caso de las enfermeras la falta de flexibilidad de horario explica una proporción adicional de varianza de intenciones de abandono. El aumento de edad explica una proporción adicional de varianza de intenciones de abandono en las auxiliares de enfermería. Las intenciones de permanencia en la organización son explicadas por la flexibilidad de horarios y autonomía en el trabajo en el caso de las enfermeras, y por menores niveles de Agotamiento Emocional y mayores niveles de Realización en el trabajo en el caso de las auxiliares de salud.
\end{abstract}

Palabras clave: Burnout; Satisfacción laboral; Autonomía laboral; Intención de abandono 


\section{INTRODUCCIÓN}

La retención de personal de enfermería y de auxiliares de salud ha sido identificado comoun problema crítico, a nivel internacional (Aiken, Sloane, Bruyneel,Vanden Heede, Sermeus \& RN4CAST Consortium, 2013; Heinen, van Achterberg, Schwendimann, Zander, Matthews, Kózka, Ensio, Sjetne, Moreno-Casbas, Ball \& Schoonhoven, 2013; Castle, Engberg, Anderson \& Men, 2007) y nacional (Salinas, Cancino, Pezoa, Salamanca \& Soto, 2007), en la implementación de programas de salud. El envejecimiento de la población, la tendencia a la movilidad laboral de las generaciones más jóvenes, y el aumento de la demanda asistencial, han sido identificados como problemas que aumentan la escasez de este tipo de profesionales y técnicos en muchas instituciones de salud. Dado lo anterior, el estudio de los antecedentes que permitan facilitar que el personal del área de salud se mantenga en sus puestos de trabajo, abandone la profesión, o se cambie de institución es considerado un tema de investigación de alta relevancia (Hayes, O’Brien-Pallas, Duffield, Shamian, Buchan, Hughes \& Stone, 2006).

\section{LITERATURA}

Múltiples variables han sido asociadas a la permanencia y a la intensión de abandono laboral de personal de salud en investigaciones previas (Aiken et al., 2013; Hayes et al., 2006). El nivel de 'burnout' ha sido consistentemente asociado a las intenciones de abandono laboral de personal de salud (Heinen et al., 2013). 'Burnout' ha sido definido como compuesto por tres dimensiones. Agotamiento Emocional con el trabajo, Despersonalización en la relación con los pacientes y Realización Personal en el trabajo (Maslach \& Jackson, 1981). La literatura previa en personal del sector salud indica que el agotamiento emocional y la despersonalización poseen una relación positiva con intención de abandono y negativas con intención de permanencia, y la Realización Personal una relación negativa con intenciones de abandono, y positiva con intención de permanencia en el puesto de trabajo (Jourdain \& Chênevert, 2010).

Además, se ha observado una relación negativa de las intenciones de abandono con la calidad del apoyo social recibido por supervisores y pares en los equipos de trabajo en muestras de enfermeras (Van der Heijden, Kümmerling, Van Dam, Van der Schoot, Estryn-Béhar \& Hasselhorn, 2010; Kim \& Stoner, 2008). Por otra parte, la satisfacción con remuneraciones y beneficios muestra resultados inconsistentes al ser asociada con intenciones de abandono y permanencia laboral (Coomber \& Barriball, 2007). Algunos estudios muestran relaciones significativas entre estas variables (Lum, Kervin, Clark, Reid \& Sirola, 1998), y otros estudios ausencia de relación estadística (Coomber \& Barriball, 2007). Es posible que las estructuras de remuneraciones de diferentes instituciones y mercados laborales generen estos resultados inconsistentes (Coomber \& Barriball, 2007). Por último, bajos grados de autonomía y control sobre el puesto de trabajo también ha sido asociado a intención de abandono de enfermeras (Cai \& Zhou, 2009; Kim \& Stoner, 2008), al igual que una excesiva carga y poca flexibilidad en los horarios de trabajo, que han sido identificados como un antecedente de intención de abandono laboral de auxiliares de enfermería (Castle et al., 2013).

En Chile los estudios sobre antecedentes de intención de abandono y permanencia laboral en el sector salud son escasos. Sin embargo, la rotación de personal en este sector es significativa. Según estadísticas de la Dirección del Trabajo (2012) el sector servicios sociales y de salud muestra una tasa de finiquitos de puestos de trabajo sobre el 13\% anual. Los costos de la deserción laboral hacen que el estudio de sus 
antecedentes sea de alta relevancia. Cuando los empleados abandonan su trabajo las organizaciones deben incurrir en costos de selección de personal, inducción en el puesto de trabajo, capacitación, además de la potencial disminución de calidad y seguridad de los cuidados que se entregan a los usuarios, y el estrés resultante en otros miembros del equipo laboral, que muchas veces deben asumir temporalmente las responsabilidades del puesto vacante (Li \& Jones, 2013). Por las razones anteriores es importante identificar los factores que pueden estar induciendo abandono laboral del personal de salud, y facilitando la permanencia de los mismos en sus puestos de trabajo. La identificación de estas variables permite intervenir en ellas e incidir en la disminución de las tasas de abandono laboral, y su consecuente impacto en la calidad y seguridad de los cuidados que se entregan, así como en la sustentabilidad de las instituciones de salud (Heinen et al., 2013).

El propósito del presente estudio es analizar, en dos instituciones de salud en Chile, la relación del nivel de 'burnout', el grado de autonomía en el puesto de trabajo, la satisfacción con remuneraciones y beneficios, la satisfacción con la flexibilidad de horarios y cargas de trabajo, y satisfacción con la calidad de las relaciones interpersonales percibidas en sus lugares de trabajo, como antecedentes que contribuyen de manera simultánea y aditiva a la intención de abandono y permanencia laboral de enfermeras y personal auxiliar de salud. Generalmente estas variables antecedentes de intención de abandono han sido estudiadas de manera aislada, y resulta de interés evaluar si la contribución de las mismas es aditiva. Este estudio contribuye a la literatura analizando la contribución simultánea de múltiples variables como predictores de intenciones de abandono laboral, y evaluando si estos predictores difieren de los antecedentes que explican la permanencia organizacional. Además, este estudio permite comparar los antecedentes de intenciones de abandono y permanencia laboral en dos categorías de trabajadores del sector salud en una muestra de Chile. Estudios similares con enfermeras son frecuentes en la literatura internacional. Sin embargo, estudios con auxiliares de enfermería son muy escasos, a pesar de ser una categoría de personal mayoritaria en las instituciones de salud.

\section{MATERIAL Y MÉTODO}

Se utiliza una metodología de cuestionarios, con un grupo de enfermeras y otro de auxiliares de enfermería, quienes evaluaron sus intenciones de abandono laboral e intenciones de permanencia laboral, así como su nivel de 'burnout', la flexibilidad de horarios de trabajo, el grado de autonomía en el puesto de trabajo, su satisfacción con la calidad de las relaciones interpersonales percibidas en su entorno laboral, y satisfacción con sus remuneraciones y beneficios. Las participantes recibieron una invitación a responder las encuestas, previa lectura y aprobación de un Consentimiento Informado, donde se ofreció confidencialidad y anonimato en el tratamiento de sus respuestas. Los procedimientos utilizados respetaron las normas éticas definidas en la Declaración de Helsinki y fueron aprobados por el Comité de Ética de la Investigación en Seres Humanos, de la Pontificia Universidad Católica de Chile.

Las muestras de enfermeras y de auxiliares de enfermería se tomaron en diferentes instituciones. Las enfermeras pertenecen a una institución hospitalaria privada de carácter docente. Un total de 192 enfermeras respondieron el cuestionario, 91.1\% de ellas mujeres. Un 59,4\% indicó tener entre 21 y 30 años, un 30,7\% entre 31 y 40 años, un $7,3 \%$ entre 41 y 50 y un $2,6 \%$ más de 50 años de edad. Las auxiliares de enfermería pertenecen a una institución de servicios de hospitalización domiciliaria privada. Las auxiliares desarrollan sus labores en los hogares de los pacientes, cumpliendo las 
indicaciones de enfermería, en una función de contacto de primera línea. Un total de 327 auxiliares de enfermería respondieron el cuestionario, $94.8 \%$ de ellas mujeres. Un $56,4 \%$ indicó tener entre 21 y 30 años, un 33,4\% entre 31 y 40 años, un 4,2\% entre 41 y 50 y un 6,0\% más de 50 años de edad.

Para medir Burnout se usaron adaptaciones al español del Inventario Burnout de Maslach efectuadas por Seisdedos (1997) y Rosales \& Cobos (2011), traducciones que se revisaron en función del instrumento original (Maslach \& Jackson, 1981), con escala de respuestas Lickert desde 0 a 6. Intensión de abandono, e intención de permanencia laboral, fueron evaluados con dos ítems de Coomber \& Barriball (2007), con escala de respuestas Lickert desde 1 a 5 . Se usaron tres preguntas de la encuesta Nursing Work Index-Revised (Aiken \& Patrician, 2000) para medir autonomía y control en el ejercicio del trabajo, con escala de respuestas Lickert desde 0 a 4 . Estas preguntas se adaptaron para ser respondidas por personal de enfermería y auxiliares. Finalmente se usaron preguntas del cuestionario de satisfacción laboral de Muller \& McClosky (1990) para evaluar la satisfacción respecto a sus remuneraciones y beneficios, la flexibilidad y carga de horario de trabajo, y con la calidad de las relaciones interpersonales, con escala de respuestas Lickert desde 0 a 4 . Por último, se controló por sexo ( $1=$ femenino, $2=$ masculino) y edad ( 21 a 30 años $=1,31$ a 40 años $=2$, 41 a 50 años=3, más de 50 años=4). Variables que en estudios anteriores han mostrado tener relación con las aquí analizadas (Aiken et al., 2013; Hayes et al., 2006). El uso de diferentes escalas Lickert de respuestas en la mayoría de los cuestionarios usados se efectuó para mitigar problemas potenciales por varianza común por error de medición (Podsakoff, MacKenzie, Lee \& Podsakoff, 2003).

\section{RESULTADOS}

Se efectuó un análisis factorial de componentes principales, con rotación Varimax, de los ítems de cada encuesta para el total de los datos del estudio. El análisis agrupó los ítems de satisfacción laboral en tres factores con Eigenvalues sobre 1,0 y con una contribución a la varianza total explicada de 53,7\%. En un primer factor se agruparon los ítems de remuneraciones y beneficios con una contribución a la varianza total de $28,2 \%$, En un segundo factor se agruparon los ítems de flexibilidad y carga de horario de trabajo con una contribución de un 14,3\%, y un tercer factor con los ítems de calidad de relaciones interpersonales con una contribución de un 11,3\%. Las cargas factoriales de los ítems fluctuaron entre 0,56 y 0,81 con un promedio de 0,71 . Los resultados de este análisis indican validez discriminante entre estos tres factores. También se efectuó un análisis factorial de componentes principales de los ítems de burnout para el total de los datos del estudio. El análisis agrupó los ítems en tres factores con eigenvalues sobre 1,0 , con una contribución a la varianza total explicada de 50,8\%. En un primer factor se agruparon los ítems de agotamiento emocional con una contribución a la varianza total de un $28,3 \%$, en un segundo factor se agruparon los ítems de despersonalización con una contribución de un 13,8\%, y un tercer factor con los ítems de realización personal con una contribución de un 8,7\%. Las cargas factoriales de los ítems fluctuaron entre 0,42 y 0,84 con un promedio de 0,68. Los resultados de este análisis indican validación de constructo de las dimensiones de burnout. En el análisis factorial de burnout dos ítems (6 y 16) originalmente pertenecientes al factor agotamiento emocional se trasladaron al factor Despersonalización, debido a las cargas factoriales de estos ítems. Este mismo traslado de estos dos ítems se efectuó en investigaciones previas con personal de salud (Poghosyan, Aiken \& Sloane, 2009). Además, un ítem de cada factor fue eliminado del estudio (ítems 12, 15 y 20) debido al 
uso de diferentes redacciones para adaptarlos a las dos muestras o por tener una muy baja carga factorial con la dimensión correspondiente. Los resultados de los análisis de componentes principales, discriminando tres factores de satisfacción laboral y tres factores de Burnout, sugieren un bajo riesgo de varianza común por error de medición.

La Tabla 1 presenta los promedios, desviación estándar, Alpha de Cronbachy correlaciones entre todas las variables del estudio para la muestra de enfermeras y auxiliares de enfermería. Los valores del Alpha de Cronbach de todas las escalas son cercanos o superan el punto de corte de 0.70 indicando una razonable consistencia interna de las escalas usadas (DeVellis, 2003). La única excepción a lo anterior es la escala de Satisfacción con remuneraciones y beneficios, que por razones conceptuales se agruparon en un índice único. Los análisis estadísticos aquí presentados no se alteran si estos dos ítems se separan.

Se efectuaron análisis de regresión múltiple para determinar la significancia de los predictores sobre la intensión de abandono organizacional. El análisis de regresión múltiple resultó significativo tanto para enfermeras $(\mathrm{R} 2=0.30, \mathrm{~F}(9 / 189)=8.73 ; \mathrm{p}<0,00)$ como auxiliares de enfermería ( $\mathrm{R} 2=0.22, \mathrm{~F}(9 / 319)=9.43 ; \mathrm{p}<0,00)$. Los resultados de regresión muestran que los predictores que explican la intención de abandono organizacional de enfermeras y auxiliares de enfermería son casi los mismos. La Tabla 2 muestra que en el caso de las enfermeras las variables de Agotamiento Emocional (burnout), Flexibilidad de horario de trabajo, Satisfacción con remuneraciones y beneficios y Grado de autonomía en el puesto de trabajo son predictores significativos. En el caso de las auxiliares de enfermería la Tabla 2 muestra que las variables de agotamiento emocional (burnout), despersonalización (burnout), satisfacción con remuneraciones y beneficios, grado de autonomía en el puesto de trabajo y edad son predictores significativos de la intención de abandono laboral. La principal diferencia, entre los determinantes de intención de abandono laboral de ambas categorías de personal, es que la flexibilidad en los horarios de trabajo, que aparece como significativo en el caso de las enfermeras, mientras que la edad es significativa en el caso de las auxiliares de enfermería.

El análisis de regresión múltiple de los predictores sobre la variable de intensión de permanencia en el trabajo actual resultó significativo tanto para enfermeras $(\mathrm{R} 2=0.18$, $\mathrm{F}(9 / 189)=4.50 ; \mathrm{p}<0,00)$ como auxiliares de enfermería $(\mathrm{R} 2=0.11, \mathrm{~F}(9 / 319)=4.36 ; \mathrm{p}<0,00)$. La Tabla 3 muestra que en el caso de las enfermeras las variables de flexibilidad de horario y grado de autonomía en el puesto de trabajo son predictores significativos de la intensión de permanencia en la organización. En el caso de las auxiliares de enfermería la Tabla 3 muestra que las variables de agotamiento emocional y realización en el trabajo son predictores significativos de la intención de permanencia en la organización. Además, las Tablas 2 y 3 muestran que no existen problemas de multicolinealidad entre los predictores en los análisis de regresión, dado que todos los índices de factor de inflación de varianza se encuentran muy por debajo del punto de corte de 10.0 sugerido por Chatterjee y Price (1991). 

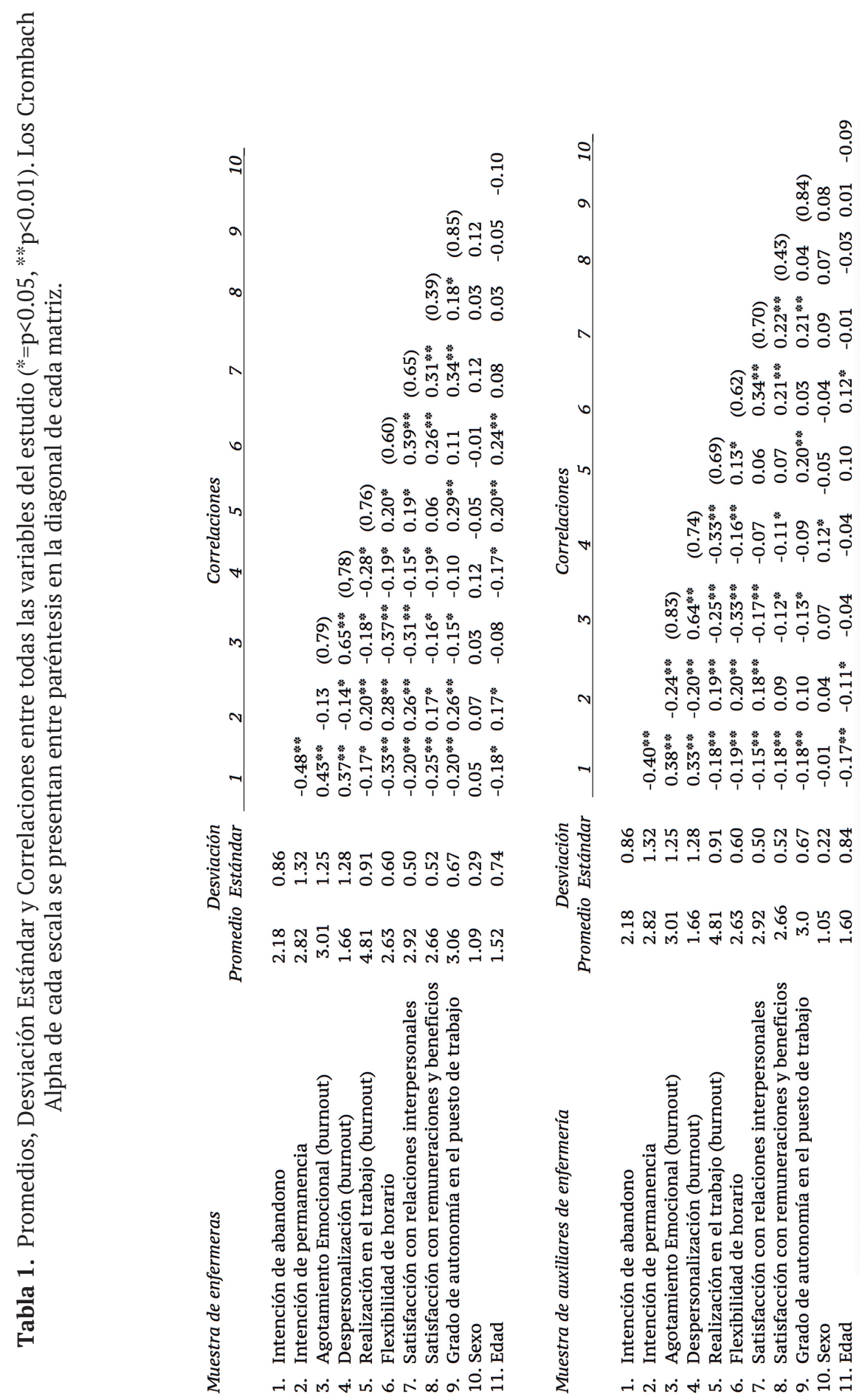


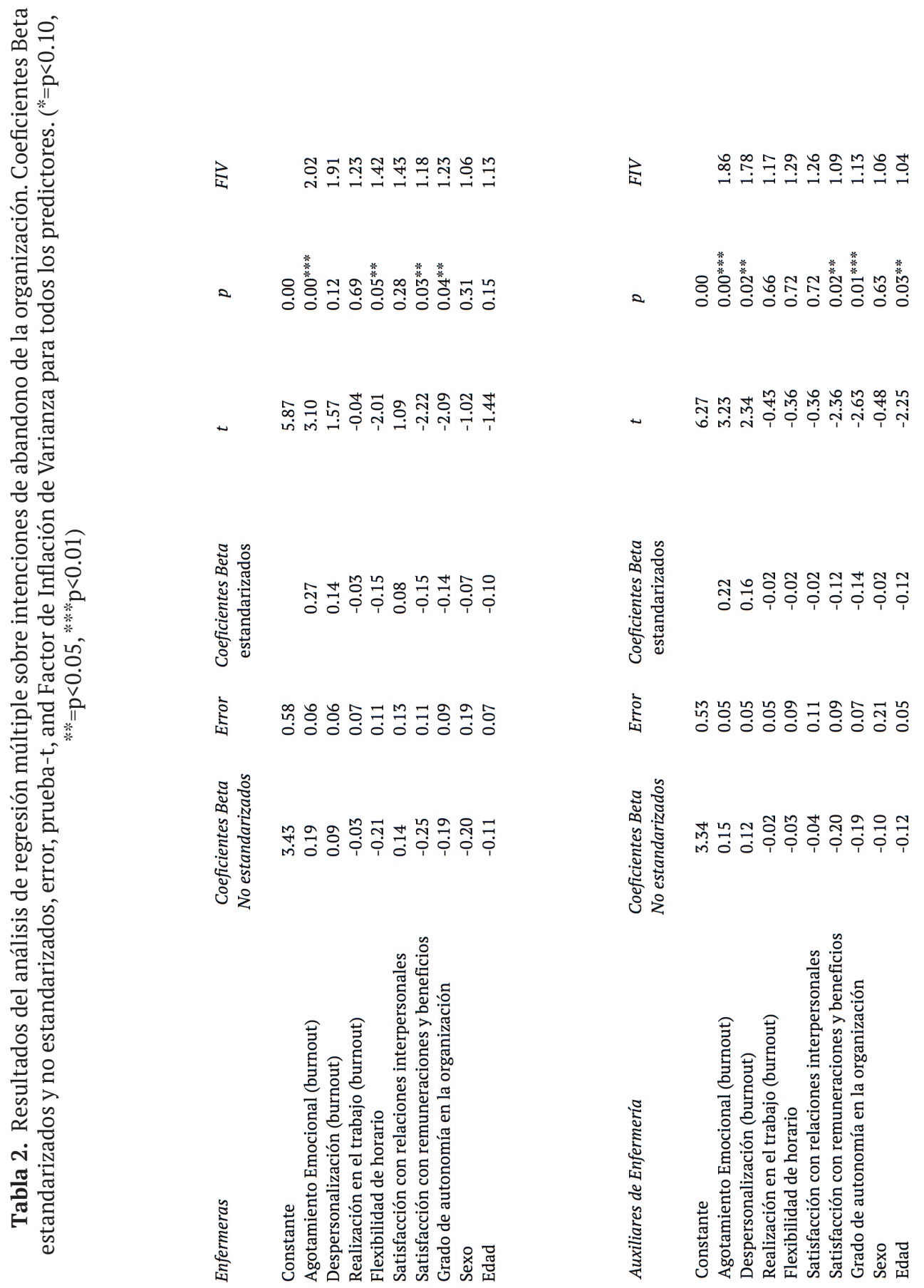




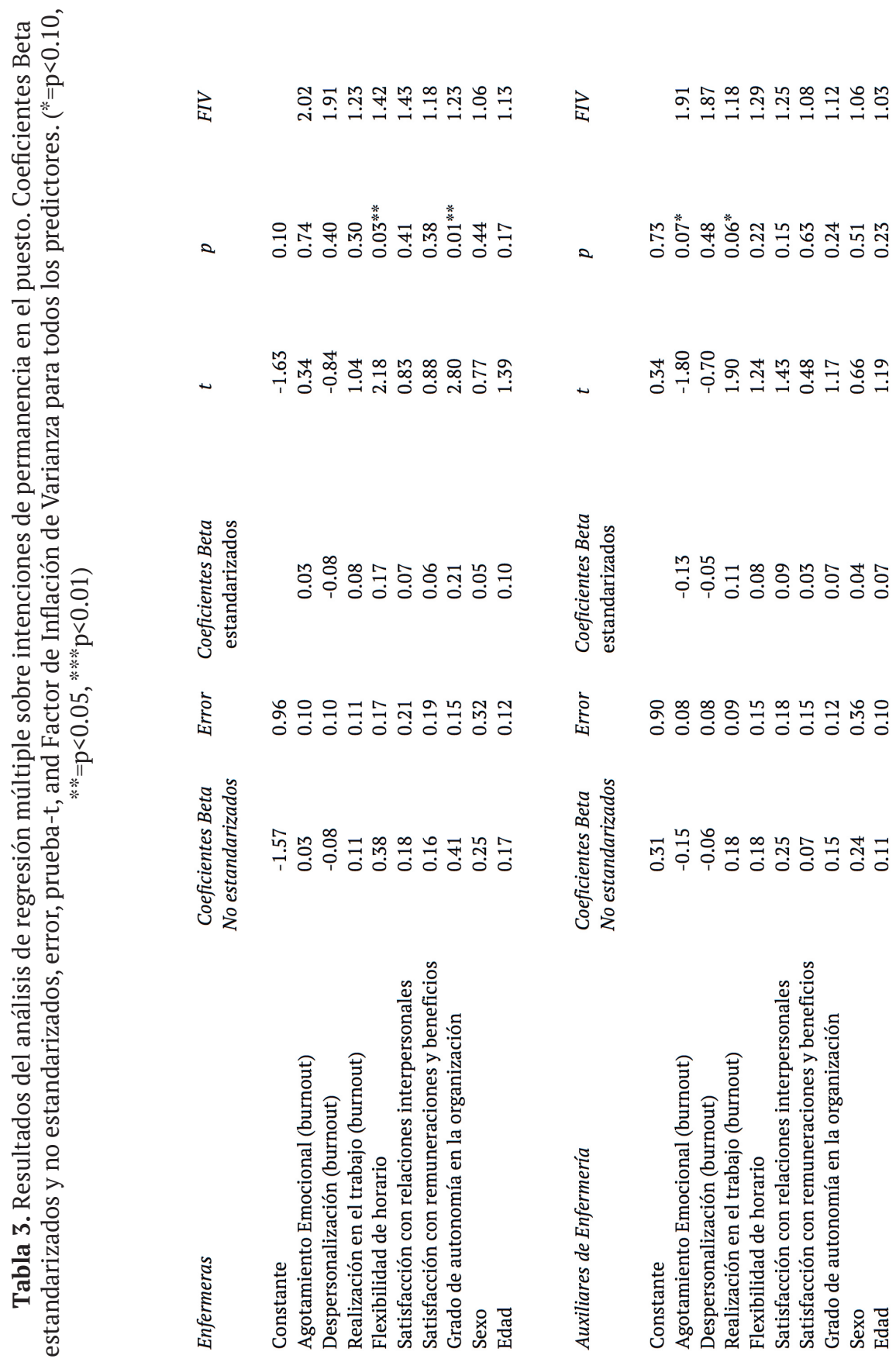




\section{DISCUSIÓN}

Este estudio contribuye a la literatura internacional sugiriendo que diferentes predictores, frecuentemente estudiados de manera aislada, aportan de manera simultánea y aditiva a explicar intención de abandono y permanencia laboral en enfermeras y auxiliares de enfermería. Desde esta perspectiva 'burnout', menores niveles de satisfacción con remuneraciones y beneficios, y menor autonomía en el trabajo explican varianza adicional de intención de abandono de ambos grupos de trabajadores. La intención de permanencia en la organización, en el caso de las enfermeras, es explicada por la presencia de Flexibilidad de Horarios y Autonomía en el trabajo, mientras que en el caso de las auxiliares de salud la intención de permanencia en la organización es explicada por menores niveles de Agotamiento Emocional y mayores niveles de Realización en el trabajo. Una posible explicación a estas diferencias en los antecedentes sobre las intenciones de permanencia de enfermeras y auxiliares podrían ser argumentada desde la perspectiva de que las enfermeras evalúan su permanencia en función de la calidad del trabajo que poseen (i.e. flexibilidad de horario y autonomía), quizás con conciencia de que hay un mercado laboral con mejores condiciones de flexibilidad de horario y autonomía. Por otra parte, las auxiliares de enfermería podrían estar evaluando su permanencia laboral desde una perspectiva del impacto del trabajo actual sobre su situación personal (Agotamiento Emocional y Realización en el trabajo actual). Si esta $u$ otras explicaciones permiten entender estas diferencias de antecedentes de intención laboral debe ser estudiado en futuras investigaciones.

Las muestras ocupadas en este estudio limitan sus conclusiones a las organizaciones particulares en que se efectuaron las evaluaciones. Por lo anterior sería de alta relevancia conducir estudios con muestras más representativas de los diferentes servicios de salud públicos y privados en Chile, para entender la magnitud del problema de retención del personal de enfermería, y auxiliares de enfermería, y las causas subyacentes de abandono laboral de las mismas. Además, el hecho de que la muestra de enfermeras y auxiliares de enfermería provengan de diferentes instituciones presenta la principal limitación para poder efectuar análisis comparativos de los resultados obtenidos entre ambos grupos de trabajadores estudiados.

En términos prácticos los resultados de este estudio contribuyen a identificar áreas de intervención para evitar abandono laboral y mejorar la retención de estas categorías de personal del sector salud. Por ejemplo, tanto en el caso de las enfermeras, como en las auxiliares de enfermería, disminuir el 'burnout', mejorar las remuneraciones y beneficios, y dar más autonomía en el trabajo, disminuyen las posibilidades de abandono laboral. Además, dar más flexibilidad de horarios de trabajo y más autonomía en el trabajo a las enfermeras ayudaría a aumentar su retención laboral. En el caso de las auxiliares de enfermería mecanismos para disminuir el agotamiento emocional y aumentar la realización en el trabajo pueden mejorar la retención laboral. La relevancia de estos resultados no solo se debe a que pueden ser de utilidad para tomar medidas que permitan disminuir las altas tasas de rotación de personal en el sector salud (Dirección del Trabajo, 2012). Además, evitar el abandono laboral y mejorar la retención, ayudaría a disminuir los costos financieros, y la calidad de las prestaciones de salud recibidas por los usuarios (Aiken et al., 2013; Li \& Jones, 
2013).

\section{REFERENCIAS}

Aiken, LH, \& Patrician, PA. (2000). Measuring organizational traits of hospitals: The Revised Nursing Work Index. Nursing research, 49(3), 146-153.

Aiken, LH, Sloane, DM, Bruyneel, L, Van den Heede, K, Sermeus, W, \& RN4CAST Consortium. (2013). Nurses' reports of working conditions and hospital quality of care in 12 countries in Europe. International Journal of Nursing Studies, 50(2), 143153.

Cai, C, \& Zhou, Z. (2009). Structural empowerment, job satisfaction, and turnover intention of Chinese clinical nurses. Nursing \& health sciences, 11(4), 397-403.

Castle, NG, Engberg, J, Anderson, R, \& Men, A. (2007). Job satisfaction of nurse aides in nursing homes: Intent to leave and turnover. The Gerontologist, 47(2), 193-204.

Chatterjee, S, and Price, B. (1991). Regression Analysis by Example. 2nd Edition. New York: John Wiley.

Coomber, B, \& Barriball, KL. (2007). Impact of job satisfaction components on intent to leave and turnover for hospital-based nurses: a review of the research literature. International journal of nursing studies, 44(2), 297-314.

DeVellis, R. F. (2003). Scale development: Theory and applications (2nd edition). Sage publications.

Dirección del Trabajo. (2012). Informe de Resultados séptima encuesta laboral (ENCLA 2011), Santiago.

Hayes, L.J., O’Brien-Pallas, L, Duffield, C., Shamian, J, Buchan, J, Hughes, F \& Stone, PW. (2006). Nurse turnover: a literature review. International journal of nursing studies, 43(2), 237-263.

Heinen, MM, van Achterberg, T, Schwendimann, R, Zander, B, Matthews, A, Kózka, M, Ensio A, Sjetne IS, Moreno-Casbas, T, Ball, J \& Schoonhoven, L. (2013). Nurses' intention to leave their profession: a cross sectional observational study in 10 European countries. International Journal of Nursing Studies, 50(2), 174-184.

Jourdain, G, \& Chênevert, D. (2010). Job demands-resources, burnout and intention to leave the nursing profession: A questionnaire survey. International Journal of Nursing Studies, 47(6), 709-722.

Kim, H, \& Stoner, M. (2008). Burnout and turnover intention among social workers: Effects of role stress, job autonomy and social support. Administration in Social Work, 32(3), 5-25.

Li, Y. \& Jones, CB. (2013). A literature review of nursing turnover costs. Journal of 
nursing management, 21(3), 405-418.

Lum, L, Kervin, J, Clark, K, Reid, F \& Sirola, W. (1998). Explaining nursing turnover intent: job satisfaction, pay satisfaction, or organizational commitment? Journal of Organizational Behavior, 19(3), 305-320.

Maslach, C \& Jackson, S. (1981). The measurement of experienced burnout. Journal of Occupational Behaviour, Vol.2 99-113.

Mueller CW, \& McCloskey JC. (1990). Nurses' job satisfaction: A proposed measure. Nursing Research, 39, 113-117.

Podsakoff, PM, MacKenzie, SB, Lee, JY, \& Podsakoff, NP. (2003). Common method biases in behavioral research: A critical review of the literature and recommended remedies. Journal of Applied Psychology, 88(5), 879-903.

Poghosyan L, Aiken LH, Sloane DM. (2009). Factor structure of the Maslach burnout inventory: an analysis of data from large scale cross-sectional surveys of nurses from eight countries. International Journal of Nurse Studies; 46(7):894-902.

Rosales Y \& Cobos D. (2011). Diagnóstico del Síndrome de Burnout en trabajadores del Centro de Inmunología y Biopreparados. Medicina y Seguridad en el Trabajo; 57 (225) 313-318.

Salinas J, Cancino A, Pezoa S, Salamanca F. \& Soto M. (2007). Vida Chile 1998-2006: resultados y desafíos de la política de promoción de la salud en Chile. Revista Panamericana de Salud Pública. 21(2/3):136-44.

Seisdedos, N. (1997). Manual MBI, Inventario Burnout de Maslach. TEA, Madrid.

Van der Heijden, BIJM, Kümmerling, A, Van Dam, K, Van der Schoot, E, Estryn-Béhar, M, \& Hasselhorn, HM. (2010). The impact of social support upon intention to leave among female nurses in Europe: Secondary analysis of data from the NEXT survey. International journal of nursing studies, 47(4), 434-44. 